\title{
Self-selection in a population-based cohort study: impact on health service use and survival for bowel and lung cancer assessed using data linkage
}

Nicola Creighton ${ }^{1 *}$ (D), Stuart Purdie ${ }^{1}$, Matthew Soeberg ${ }^{2}$, Richard Walton ${ }^{1}$, Deborah Baker ${ }^{1,3}$ and Jane Young ${ }^{4}$

\begin{abstract}
Background: In contrast to aetiological associations, there is little empirical evidence for generalising health service use associations from cohort studies. We compared the health service use of cohort study participants diagnosed with bowel or lung cancer to the source population of people diagnosed with these cancers in New South Wales (NSW), Australia to assess the representativeness of health service use of the cohort study participants.

Methods: Population-based cancer registry data for NSW residents aged $\geq 45$ years at diagnosis of bowel or lung cancer were linked to the 45 and Up Study, a NSW population-based cohort study (N 267,000). We measured hospitalisation, emergency department (ED) attendance and all-cause survival, and risk factor associations with these outcomes using administrative data for cohort study participants and the source population. We assessed bias in prevalence and risk factor associations using ratios of relative frequency (RRF) and relative odds ratios (ROR), respectively.

Results: People from major cities, non-English speaking countries and with comorbidites were under-represented among cohort study participants diagnosed with bowel $(n=1837)$ or lung $(n=969)$ cancer by $20-50 \%$. Cohort study participants had similar hospitalisation and ED attendance compared with the source population. One-year survival after major surgical resection was similar, but cohort study participants had up to $25 \%$ higher post-diagnosis survival (lung cancer 3-year survival: RRF $=1.24,95 \%$ confidence interval 1.12,1.37). Except for area-based socioeconomic position, risk factors associations with health service use measures and survival appeared relatively unbiased.

Conclusions: Absolute measures of health service use and risk factor associations in a non-representative sample showed little evidence of bias. Non-comparability of risk factor measures of cohort study participants and non-participants, such as area-based socioeconomic position, may bias estimates of risk factor associations. Primary and outpatient care outcomes may be more vulnerable to bias.
\end{abstract}

Keywords: Cohort studies, Selection bias, Health care utilisation, Cancer, Sociodemographic factors

\footnotetext{
* Correspondence: Nicola.Creighton@health.nsw.gov.au

${ }^{1}$ Cancer Institute NSW, PO Box 41, Alexandria, Sydney, NSW 1435, Australia

Full list of author information is available at the end of the article
}

(c) The Author(s). 2018 Open Access This article is distributed under the terms of the Creative Commons Attribution 4.0 International License (http://creativecommons.org/licenses/by/4.0/), which permits unrestricted use, distribution, and reproduction in any medium, provided you give appropriate credit to the original author(s) and the source, provide a link to the Creative Commons license, and indicate if changes were made. The Creative Commons Public Domain Dedication waiver (http://creativecommons.org/publicdomain/zero/1.0/) applies to the data made available in this article, unless otherwise stated. 


\section{Background}

Cohort studies have been established around the world to examine health and health care use in ageing populations [1-8]. Applying findings from these cohorts to health service policy and practice is vital to realising the public health benefit of these studies. Participants in cohort studies are typically healthier and more socioeconomically advantaged than the general population by self-selection or design (e.g. British Doctors Study). As a result, the prevalence of exposures and absolute risk of disease or death among cohort study participants are often different to their source population. While the basis for generalising aetiological associations from non-representative cohorts is well established [9], there is little empirical evidence for generalising health service use associations. Additionally, absolute measures of health service use are often required to inform health service policy and practice. The few studies examining the effect of self-selection on absolute measures of health service use are conflicting, finding higher $[10,11]$ and lower $[12$, 13] health service use among participants.

The 45 and Up Study is a population-based cohort study in New South Wales (NSW), Australia, that was established to improve knowledge of ageing, with health service use a priority area [14]. Cancer, a major ageing-associated disease, is the largest cause of burden of disease in Australia [15], is among leading causes in other high-income countries and is becoming a significant burden in middle- and low-income countries [16]. Providing effective, efficient and equitable access to cancer care services is important in reducing this burden. However, there is evidence that patterns of health service use, such as late diagnosis and reduced treatment uptake, across population subgroups lead to poorer cancer outcomes [17-19]. Demonstrating that patterns of health service use and associations with risk factors among cohort study participants are generalisable to the source population enables research findings from cohort studies to be applied with more confidence to health service policy and practice.

In this study, we aimed to assess differences in inpatient hospital use, emergency department attendance and survival among 45 and Up Study participants diagnosed with lung or bowel cancer compared with people diagnosed with these cancers aged 45 years and older in the NSW population using linked population-based cancer registry and administrative health data. We compared estimates of associations between risk factors (remoteness of residence, socioeconomic position, country of birth, comorbidity) and health service use and survival outcomes to assess selection bias.

\section{Methods}

\section{Study design}

This study used de-identified linked cancer registry, administrative hospital, death registry and 45 and Up Study cohort data. Around 267,000 NSW residents aged 45 years and older joined the Sax Institute's 45 and Up Study between February 2006 and December 2009, representing around $10 \%$ of this age group. Participants were randomly selected from the Department of Human Services (formerly Medicare Australia) enrolment database, a national publicly funded universal health care scheme. People aged 80 years and older and those from rural areas were over-sampled by a factor of two and all remote residents were sampled. Participants were recruited by completing a postal questionnaire and consenting to follow-up and linkage of their health-related records. The response rate was reported as $18 \%$ mid-recruitment period [14] and additionally participants $(<1 \%)$ volunteered via a hotline.

Cancer case data were obtained from the NSW Cancer Registry (NSWCR), a statutory registry of all invasive cancer cases (excluding non-melanoma skin cancer) diagnosed in NSW residents. Admission records for all NSW public and private hospitals were obtained from the NSW Admitted Patient Data Collection. Emergency department (ED) attendances at public hospitals were obtained from the NSW Emergency Department Data Collection, which had substantively complete coverage of EDs in metropolitan areas but was incomplete for regional areas for the study period. Attendance data were not available for the small number $(<5)$ of EDs at private hospitals which made up $<5 \%$ of ED activity during the study period $[20,21]$. Mortality follow-up was from deaths recorded on the NSW Registry of Birth Deaths and Marriages.

The study was conducted with ethical approval from the NSW Population and Health Services Research Ethics Committee (HREC/14/CIPHS/60). Probabilistic linkage of the datasets was conducted by the Centre for Health Record Linkage (CHeReL) with an estimated false positive rate of 5 per 1000 (www.cherel.org.au). Identifying information (such as names and addresses) was separated from content information in the datasets to protect privacy. The CHeReL uses Choicemaker software to match identifiers and create a de-identified Project Person Number that enables records for an individual to be ascertained across the study datasets by researchers without accessing identifying information. The 45 and Up Study is approved by the University of New South Wales Ethics Committee.

\section{Study population}

People aged $\geq 45$ years diagnosed with bowel cancer (International Classification of Diseases, 10th Edition, Australian Modification [ICD-10-AM] C18-C20) or non-small cell lung cancer (ICD-10-AM C34, excluding m8041-m8045 and m8246; hereafter 'lung cancer') between February 2006 (commencement of 45 and Up Study recruitment) and December 2012 (the most recent data available at the time of extraction) were ascertained 
from the NSWCR. Bowel and lung cancer were selected since they are commonly diagnosed cancers, are leading causes of cancer death and have high rates of health service use in Australia [22]. People with a cancer diagnosed prior to the index cancer (from January 2000 onwards) or with another cancer case diagnosed within three months of the index cancer were excluded. Cases of an uncommon histology type, notified to the NSWCR by death certificate only, or with an unknown diagnosis date or place of residence were excluded. Cancers with uncommon histology types were excluded since they have different treatment patterns and outcomes.

\section{Outcomes and study variables}

We examined health service use in the year prior to and the year after diagnosis. We used measures of hospital use (number of overnight admissions and number of weeks in hospital, excluding hospitals that primarily provide sub- and non-acute care) and ED attendance since linked population data are available for these areas of health service use. We measured major resection (defined by the Australian Classification of Health Interventions) since surgery is the main curative treatment for bowel and lung cancer. We measured all-cause one- and three-year post-diagnosis survival and, for those who underwent resection, one-year post-operative survival. Survival outcomes were measured since there are high rates of health service use in the lead up to death [23]. Mortality follow-up was to September 2016.

Age at diagnosis, sex, area-based socioeconomic position (Index of Relative Socioeconomic Disadvantage [24] for Census Districts), remoteness of residence [25] and extent of disease at diagnosis were obtained from the NSWCR. Country of birth was obtained from the NSWCR for people diagnosed between 2006 and 2010 but was unavailable from the NSWCR for 2011-2012. Country of birth was obtained from hospital admission records for this period. Hospital type (public or private), urgency of admission and the Charlson comorbidity score [26] (calculated with a five year look-back from hospital-recorded diagnoses) were obtained from hospital admission records.

\section{Analysis}

We compared demographic, cancer case and health service use characteristics by 45 and Up Study participation status using a ratio of relative frequency (RRF). This was calculated by dividing the proportions in the 45 and Up Study by the proportion in the NSW cancer population for each categorical variable $[27,28]$. A ratio greater than one indicates over-representation and a ratio below one indicates under-representation among 45 and Up Study participants. We restricted the examination of risk factor associations to resection use, one-year post-diagnosis survival, $>4$ weeks in hospital and $>2 \mathrm{ED}$ attendances in the year after cancer diagnosis. We focused on examining potential bias in associations with remoteness of residence, socioeconomic position, country of birth and comorbidity since these factors are often the focus of health service use studies. Associations with these factors were examined using a multivariable logistic regression model including all the factors of interest and adjusting for factors with known prognostic importance. In the model of resection status, sex, age and extent of disease at diagnosis were included as prognostic factors. In the models of the other outcomes, resection status was included as an additional prognostic factor. Adjusted relative odds ratios (RORs) were calculated as the ratio of the OR of 45 and Up Study participants to the OR of the NSW cancer population. Confidence limits (CLs) for the RRFs and RORs were calculated using the formula described by Nohr et al. [28]. The formula assumes the subsample is a random sample of the population, which is not the case here; however, the coverage properties were found to be adequate in a similar study [28].

\section{Results}

Demographic and cancer characteristics

A total of $233,133 \mathrm{NSW}$ residents aged $\geq 45$ years were diagnosed with 245,266 cancer cases between February 2006 and December 2012 (Fig. 1). In NSW in 2008-12, the incidence per 100,000 age-standardised to the world population was 44.8 and 33.3 among men and 31.5 and 21.6 among women for bowel cancer and lung cancer respectively for all ages [29]. A total of 17,661 participants of the 45 and Up Study were diagnosed with cancer after enrolment, $7.6 \%$ of all NSW residents diagnosed. Lung cancer was under-represented among 45 and Up Study participants diagnosed with cancer $(7.8 \%[n=1379]$ v $10.1 \%[n=23,537] ; \mathrm{RRF}=0.77, \mathrm{CL}$ $0.74,0.81)$. In the final analysis cohorts, $6.8 \%(n=1837)$ and $5.6 \%(n=969)$ of NSW residents aged $\geq 45$ years at diagnosis of bowel or lung cancer, respectively, were 45 and Up Study participants.

Sex and age distributions of 45 and Up Study participants diagnosed with bowel or lung cancer were similar to the NSW cancer population distributions (Table 1). Although there was under-representation of the youngest age groups, the median and interquartile ranges of age at diagnosis were similar. People from regional and remote areas were over-represented among 45 and Up Study participants by up to $50 \%$. The distribution of socioeconomic position was similar between participants and the source population, particularly for bowel cancer. However, the over-representation of regional and remote areas among 45 and Up Study participants affects the distribution of socioeconomic position since these areas are generally more socioeconomically disadvantaged 


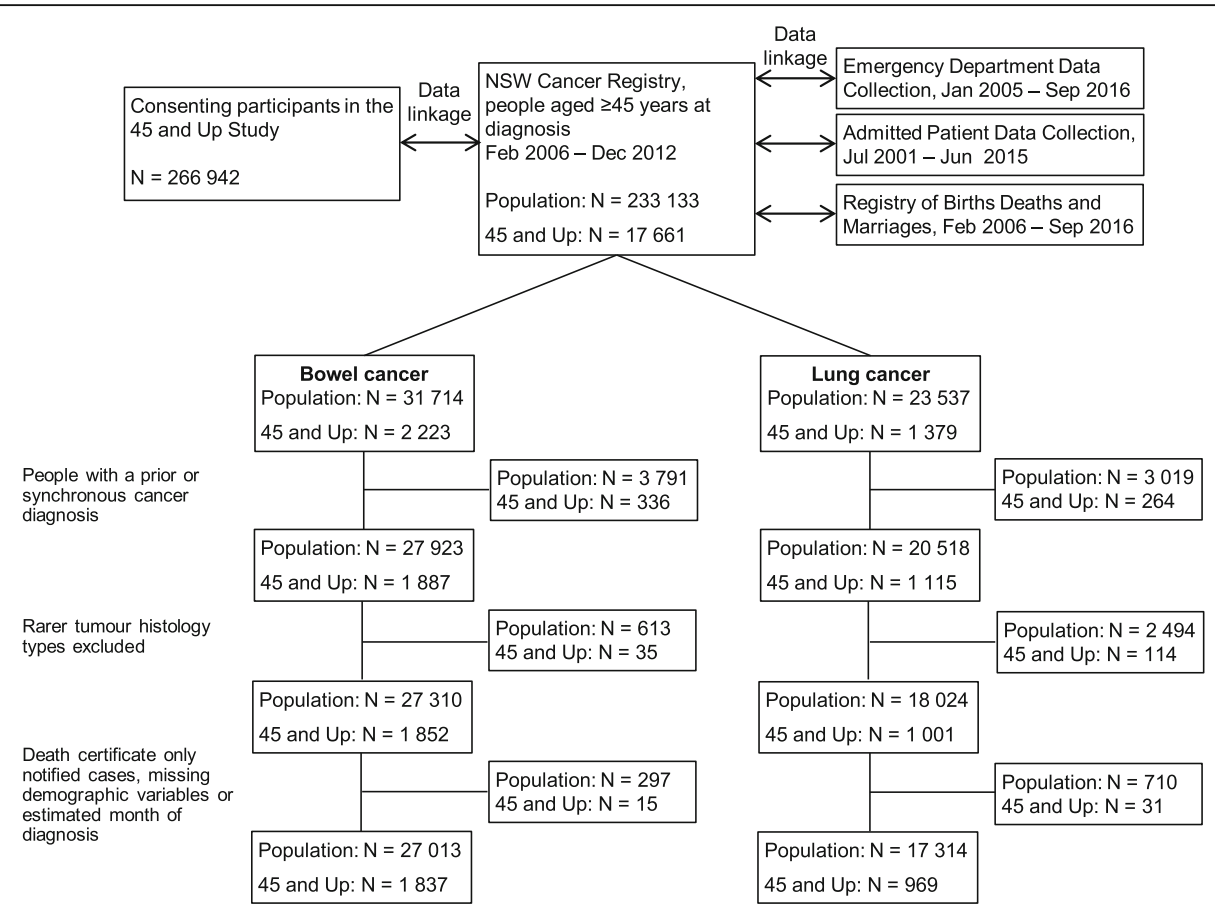

Fig. 1 Study cohort

than major cities. Stratifying by remoteness, the overrepresentation of people from less disadvantaged areas was evident. For example in major cities, people diagnosed with bowel cancer from areas in the least disadvantaged socioeconomic quintile were over-represented in the 45 and Up Study (26.7\% [ $n=248]$ v $21.9 \%$ [ $n=$ 3918]; RRF 1.22, CL 1.10, 1.35) and the most disadvantaged quintile was under-represented $(14.2 \%[n=132] \mathrm{v}$ 19.3\% [ $n=3452$ ]; RRF 0.74, CL 0.63, 0.86) (see Additional file 1: Table 1). People from non-English speaking countries of birth were under-represented by a factor of two for both cancers. Extent of disease at diagnosis was similar, although the proportion of 45 and Up Study participants diagnosed with localised bowel cancer was slightly higher $(34.7 \%[n=637]$ v $31.4 \%$ [ $n=8493$ ]; RRF 1.10, CL 1.04, 1.17). 45 and Up Study participants had lower Charlson comorbidity scores for both cancers, with a higher proportion of 45 and Up Study participants with a score of zero and a lower proportion with a score of two or more.

\section{Hospital use, ED attendance and survival}

Hospital use and ED attendance in the year prior to bowel or lung cancer diagnosis were similar for 45 and Up Study participants compared with the NSW bowel and lung cancer populations (Table 2). In the year after diagnosis, the number of hospital admissions and weeks in hospital were similar, although slightly fewer (RRF 0.9) 45 and Up Study participants spent more than four weeks in hospital and a higher proportion of stays were in private hospitals. A higher proportion of 45 and Up Study participants had no ED attendances in the year after diagnosis, which was also the case for residents of major cities where coverage of ED attendances was complete (not shown). Emergency bowel resections were under-represented among 45 and Up Study participants (12.5\% [ $n=185]$ v $15.6 \%$ [ $n=3324]$; RRF 0.80, CL 0.70, 0.92 ). One year post-operative survival was similar for 45 and Up Study participants for both cancers (Table 3). One-year post-diagnosis survival was higher among 45 and Up Study participants by two and six percentage points for bowel and lung cancer respectively. Three-year post-diagnosis survival was around five percentage points higher among 45 and Up Study participants for both cancers, which for lung cancer is $24 \%$ higher than the population value $(26.4 \%[n=256] \mathrm{v}$ $21.3 \%[n=3686]$; RRF 1.24 , CL 1.12, 1.37).

\section{Associations between risk factors and outcomes}

There was little evidence of systemic bias in the estimates of associations between health service use and survival outcomes and remoteness of residence, country of birth and Charlson comorbidity score with odds ratios generally in the same direction and of similar magnitude among 45 and Up Study participants and the NSW population (Figs. 2 and 3). However, there are examples of odds ratios for 45 and Study participants and the NSW population being in the opposite direction for 
Table 1 Cancer case characteristics of people diagnosed with bowel or lung cancer and ratio of relative frequency (RRF), 45 and Up Study participants and NSW residents aged $\geq 45$ years

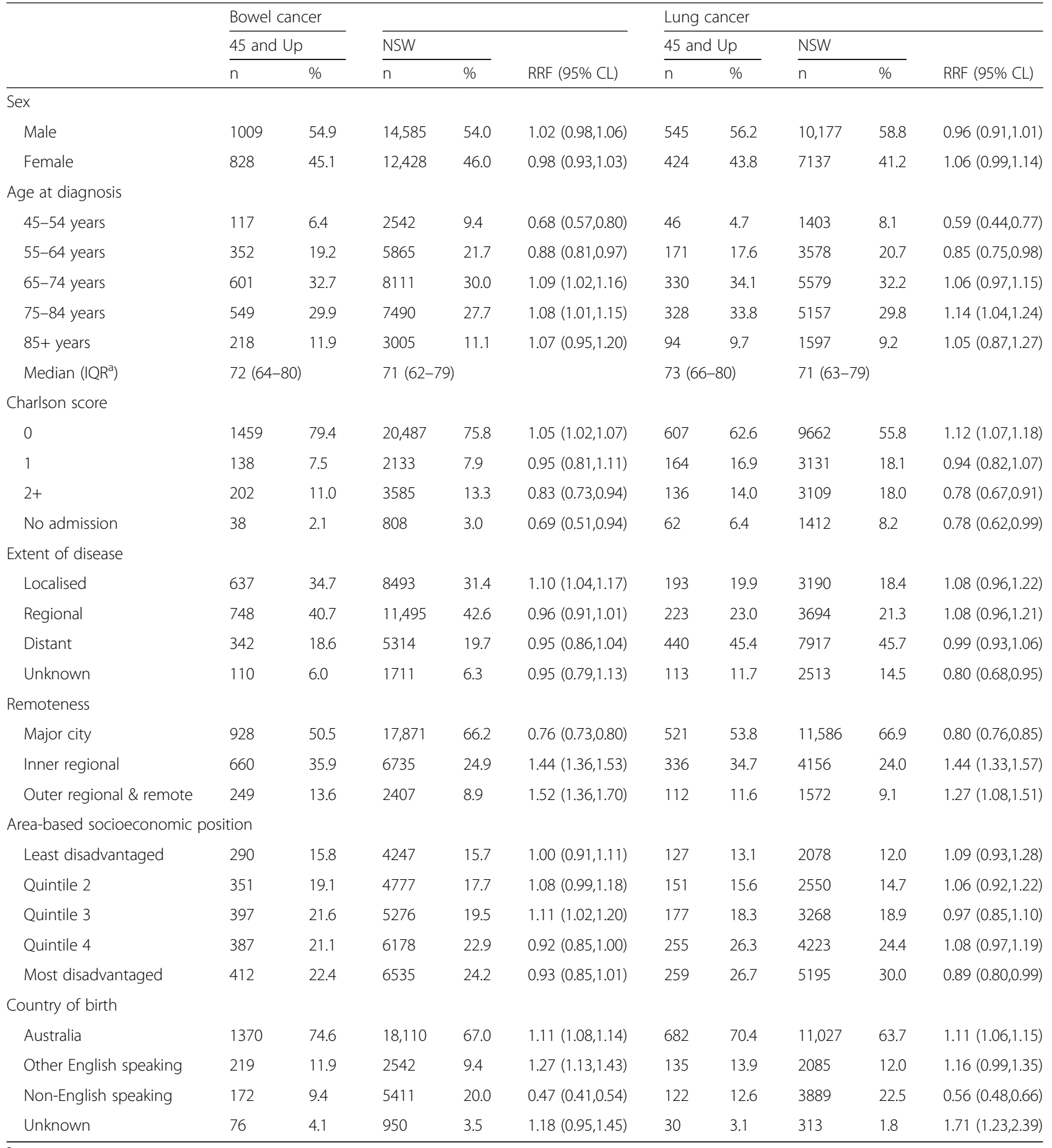

${ }^{a} \mathrm{IQR}=$ interquartile range

$C L$ confidence limits

people born in non-English speaking countries (odds of $>4$ weeks in hospital and surviving one year after diagnosis of bowel cancer) and for people from outer regional and remote areas (odds of surviving one year after diagnosis of bowel cancer). There are examples of the magnitude of odds ratios for 45 and Study participants and the NSW population differing (lower odds of $>4$ weeks in hospital for Charlson score of $2+$ and higher odds of $>2$ ED attendances for Charlson score of 1 for 45 and Up Study participants diagnosed with lung 


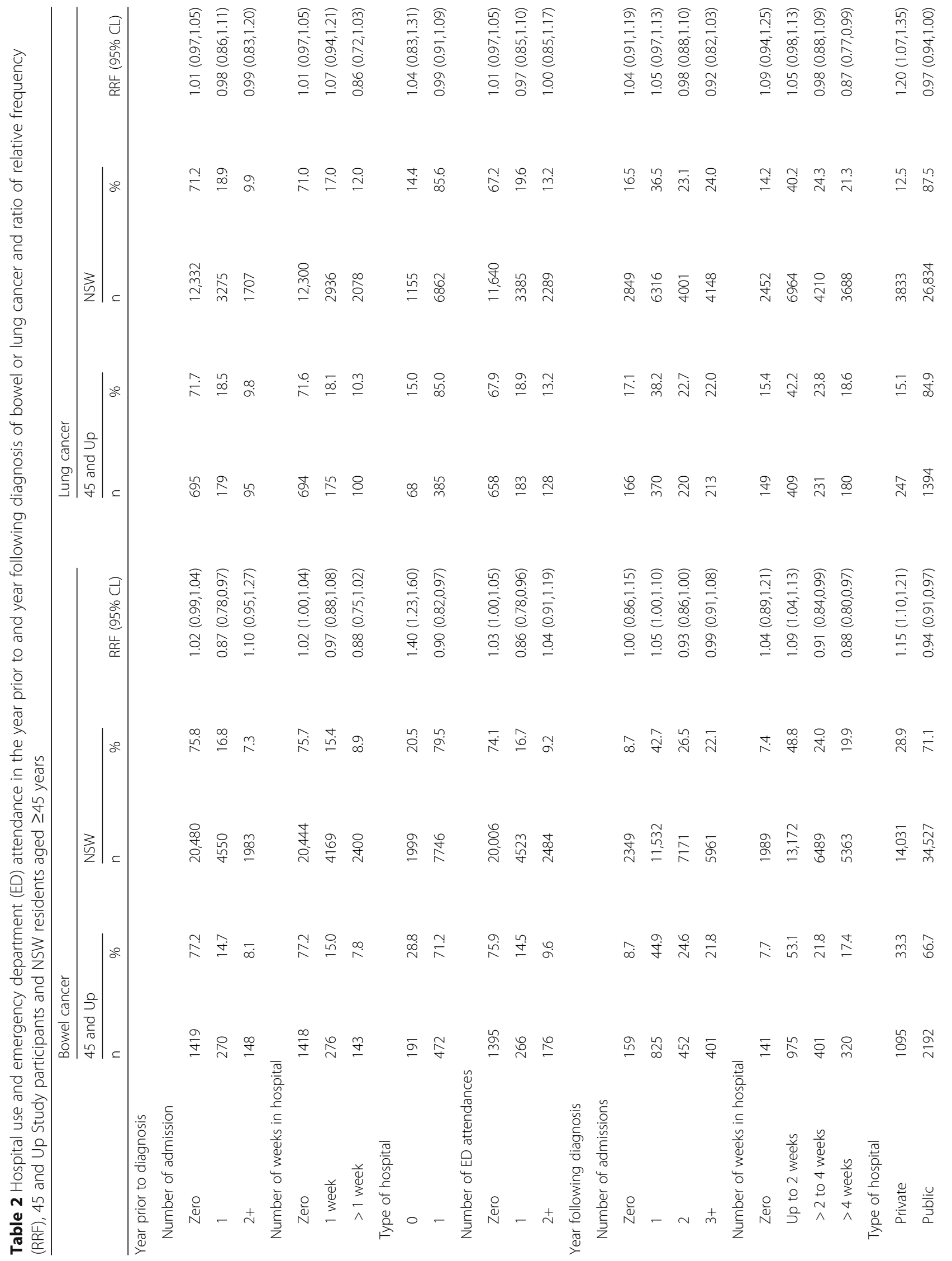




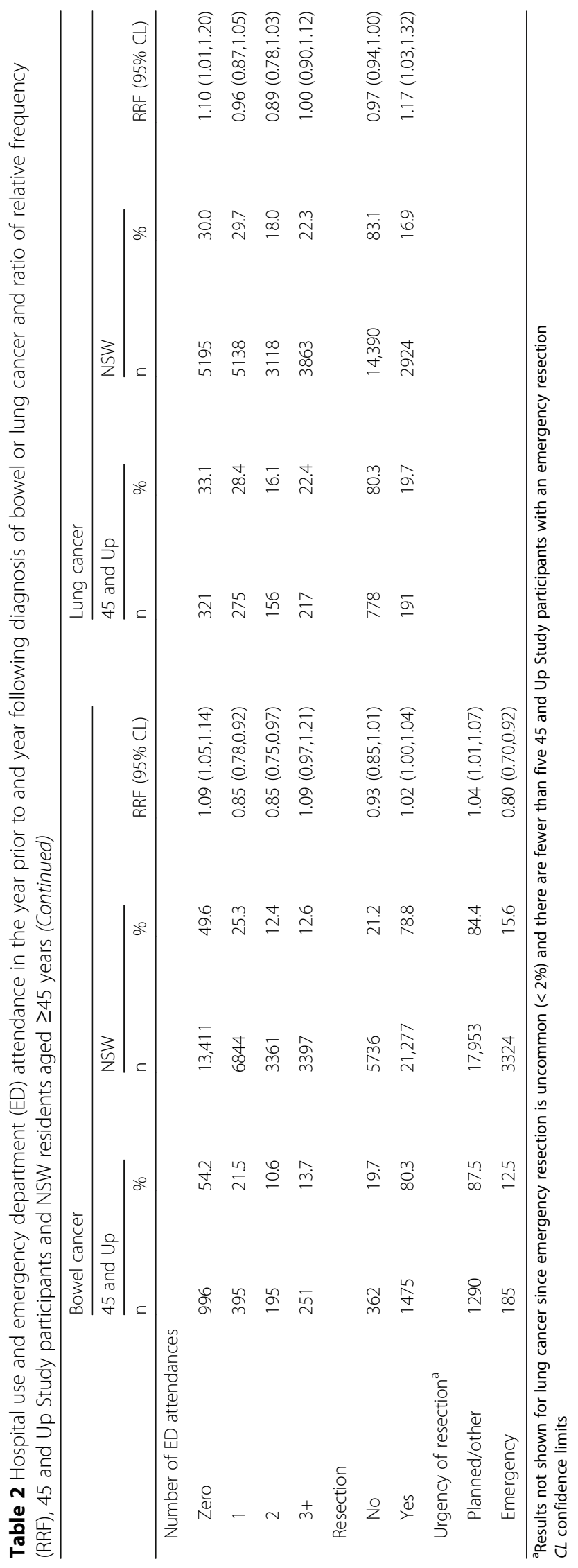


Table 3 Survival outcomes following diagnosis of bowel or lung cancer and ratio of relative frequency (RRF), 45 and Up Study participants and NSW residents aged $\geq 45$ years

\begin{tabular}{|c|c|c|c|c|c|c|c|c|c|c|}
\hline & \multicolumn{5}{|c|}{ Bowel cancer } & \multicolumn{5}{|c|}{ Lung cancer } \\
\hline & \multicolumn{2}{|c|}{45 and Up } & \multicolumn{2}{|l|}{ NSW } & \multirow[b]{2}{*}{ RRF $(95 \% \mathrm{CL})$} & \multicolumn{2}{|c|}{45 and Up } & \multicolumn{2}{|l|}{ NSW } & \multirow[b]{2}{*}{ RRF $(95 \% \mathrm{CL})$} \\
\hline & $n$ & $\%$ & $n$ & $\%$ & & $n$ & $\%$ & $n$ & $\%$ & \\
\hline \multicolumn{11}{|c|}{ One-year post-operative survival } \\
\hline No & 140 & 9.5 & 2258 & 10.6 & $0.89(0.77,1.04)$ & 25 & 13.1 & 366 & 12.5 & $1.05(0.72,1.52)$ \\
\hline Yes & 1335 & 90.5 & 19,019 & 89.4 & $1.01(0.99,1.04)$ & 166 & 86.9 & 2558 & 87.5 & $0.99(0.87,1.14)$ \\
\hline \multicolumn{11}{|c|}{ One-year post-diagnosis survival } \\
\hline No & 273 & 14.9 & 4646 & 17.2 & $0.86(0.78,0.96)$ & 506 & 52.2 & 10,082 & 58.2 & $0.90(0.85,0.95)$ \\
\hline Yes & 1564 & 85.1 & 22,367 & 82.8 & $1.03(1.01,1.05)$ & 463 & 47.8 & 7232 & 41.8 & $1.14(1.07,1.22)$ \\
\hline \multicolumn{11}{|c|}{ Three-year post-diagnosis survival } \\
\hline No & 508 & 27.7 & 8716 & 32.3 & $0.86(0.80,0.92)$ & 713 & 73.6 & 13,628 & 78.7 & $0.93(0.90,0.97)$ \\
\hline Yes & 1329 & 72.3 & 18,297 & 67.7 & $1.07(1.04,1.10)$ & 256 & 26.4 & 3686 & 21.3 & $1.24(1.12,1.37)$ \\
\hline
\end{tabular}

cancer). Multivariable adjustment did not substantially change odds ratio estimates for risk factors (see Additional file 1: Tables).

In the NSW population, greater socioeconomic disadvantage was associated with lower odds of resection and one-year survival for bowel cancer whereas there was little evidence of an effect among 45 and Up Study participants from the point estimates of the disadvantage quintiles, although confidence intervals were wide. Odds of $>2$ ED attendances in the year after bowel cancer diagnosis were in the same direction for 45 and Up Study participants as for the NSW population but were consistently around 1.5 times higher for the disadvantage quintiles. The relative consistency of differences in the magnitude and direction of odds ratio estimates for socioeconomic position across multiple outcomes among 45 and Up Study participants with bowel cancer could be indicative of bias.

\section{Discussion}

The expectation of cohort study participants being healthier and wealthier than their source population was met in regard to health but was not as straightforward for wealth. The marginal distribution of socioeconomic position of 45 and Up Study participants diagnosed with bowel or lung cancer was similar to the source population of people aged $\geq 45$ years diagnosed with these cancers. However, the expected over-representation of people from more socioeconomically advantaged areas was evident when stratified by remoteness. We attribute the difference between the stratified and marginal distributions of socioeconomic position to the over-representation of people from regional and remote areas. People from regional and remote areas were over-sampled in the design of 45 and Up Study to facilitate examining effects of rurality [14] and these areas are generally more socioeconomically disadvantaged than major cities [24].
Slightly more 45 and Up Study participants diagnosed with bowel or lung cancer had no comorbidity, with participants having higher post-diagnosis survival compared with the population. Lung cancers were less common among 45 and Up Study participants compared to the NSW population. Since most lung cancers are smoking-related [30], this likely reflects the lower prevalence of smokers and greater proportion of never smokers in the 45 and Up Study compared to NSW population survey-based estimates at baseline (7.4 and 12\% smoking prevalence [31], 56\% [32] and 40-50\% never smokers [33] in the 45 and Up Study and NSW population respectively). A higher proportion of 45 and Up Study participants were diagnosed with localised bowel cancer compared to the NSW population, which may be related to 45 and Up Study participants having higher rates of bowel screening compared to NSW population estimates [31]. A national government-funded screening program was phased in from late 2006 to facilitate early detection of bowel cancer. Additionally screening tests have been available from pharmacies and medical practitioners. In contrast, lung cancer does not have a screening program and the diagnosis of localised lung cancer was similar between 45 and Up Study participants and the NSW population. Despite these differences, absolute measures of hospital and emergency department use in the year prior to and after cancer diagnosis were similar to the population estimates.

Estimates of risk factor associations among 45 and Up Study participants were generally consistent with population estimates, despite participants not being a representative sample in terms of these factors. While this is a demonstration of representativeness not being required for associations to be generalisable, the converse, that representativeness does not guarantee generalisability, was also demonstrated. The only risk factor that showed evidence of systemic bias was socioeconomic position among 


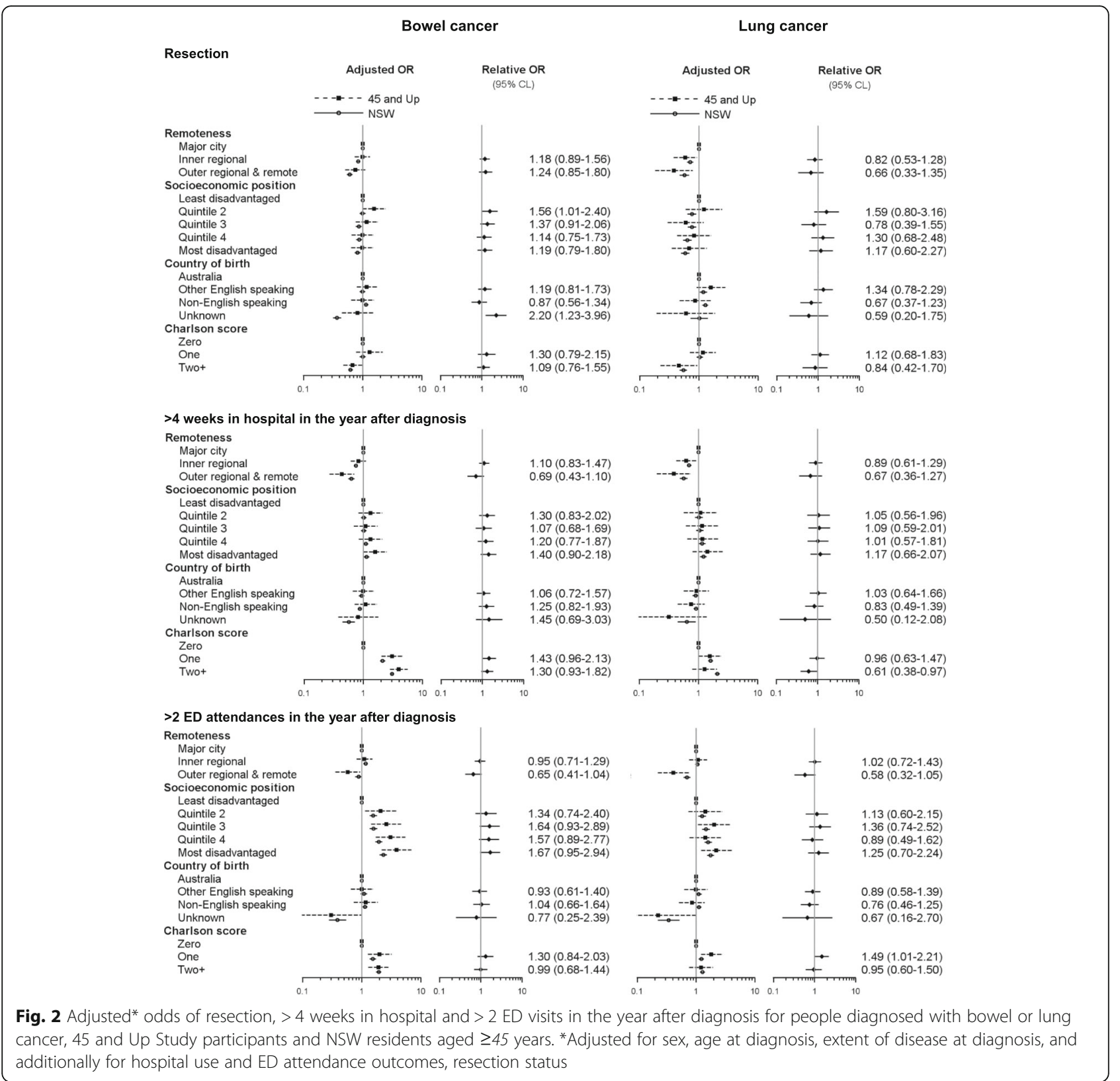

people with bowel cancer, which had a similar marginal distribution to the population. This apparent bias may in part be due to differing effects of socioeconomic disadvantage on health care utilisation in urban and rural settings. As in most epidemiological studies, the measure of socioeconomic position used in this study is a general index that may not capture contextual effects of disadvantage in urban and rural settings [24, 34]. The apparent bias may also be due to the area-level measure of socioeconomic position used in this study since an individual-level measure was not available in the population cancer data. 45 and Up Study participants may have different individual-level socioeconomic characteristics to those in the same area, making participants not comparable to non-participants. Similarly, since the 45 and Up Study baseline questionnaire was only available in English, country of birth associations were measured among people with sufficient English proficiency to respond which could have contributed to instances of non-English speaking country of birth associations being in the opposite direction to the population estimates.

Selection bias can occur when there are joint risk factors for study participation and outcomes and, furthermore, the magnitude of bias depends on the strength of these associations [35]. Health service use studies may be prone to selection bias since factors such as health 


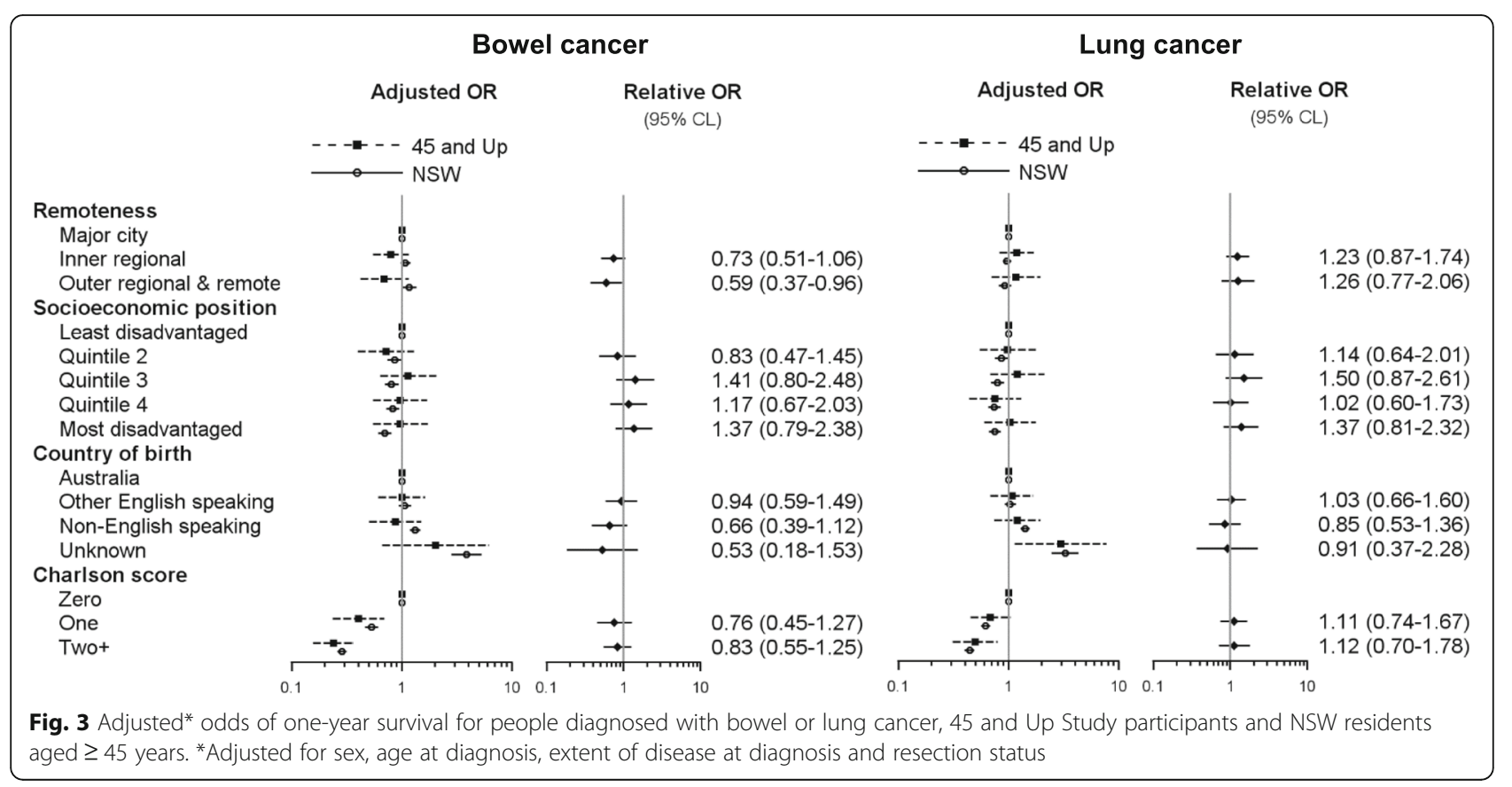

literacy and health-seeking behaviours are likely to be associated with participation in a cohort study and are associated with health service use [36, 37]. Selection bias can be minimised by including factors associated with selection and outcome in adjustment models [35]. However, there are no questions on health literacy and few questions on health-seeking behaviours in the 45 and Up Study. It would be beneficial for cohort studies established with an aim of examining health service use to include validated measures of health literacy and health-seeking behaviours.

In aetiological studies, a key consideration in assessing the generalisability of associations is whether the underlying biological mechanisms are the same in participants and non-participants [38]. In health service use studies, non-biological mechanisms such as attitudes and beliefs towards health service use also need to be considered. In other studies, hospital use by responders to a health survey was similar to non-responders but out-of-hospital health service use differed. $[10,11]$ Hospital use is potentially less likely to be impacted by a person's health-seeking propensity than out-of-hospital care since admitting physicians act as gatekeepers. Much activity for the early detection and diagnosis of cancer occurs in the primary care and outpatient settings. Health service use in response to cancer symptoms depends not only on clinical factors, but also psychosocial factors such as knowledge of symptoms and fear of cancer [39, 40]. Population-level primary care and outpatient data are not available for linkage studies in NSW. Health service use in these settings may be more vulnerable to the impacts of self-selection and requires further examination.
With the large number of comparisons in this study, some differences between estimates from 45 and Up Study participants and the NSW population are likely to occur by chance. Additionally, the 45 and Up Study participants were a small sample of the population and differences could result from sampling error rather than non-sampling error such as self-selection. The precision of the study estimates was limited by the small number of 45 and Up Study participants diagnosed with cancer. For individual cancer sites, even large cohort studies may be underpowered for the detection of differences between risk groups for health service use outcomes [41]. Furthermore, small numbers can reduce the number of confounders able to be included in adjustment models due to sparse-data bias [42]. The number of cancer cases diagnosed among 45 and Up Study participants will increase with longer follow-up. However, the findings of health service use studies using cancer cases diagnosed over long time periods may have limited applicability to health service policy and practice which often require timely data.

There are few studies examining the impact of self-selection on health service use outcomes and none focusing on cancer that we are aware of. Of these studies, most have examined participation in surveys with response rates of $50-80 \%$ conducted in Scandinavia or the Netherlands with one US Study [10, 11, 13, 43, 44]. The effect of self-selection on hospitalisation and psychiatric care has been examined in one cohort study [12] which had participation rates of $65-90 \%$ compared with the 45 and Up Study (18\%) [14]. These studies have focussed on absolute measures of health service use and have 
reported both higher [10, 11, 44] and lower [12, 13, 43] health service use among participants. One study reported that health service use was only slightly (3-6\%) lower among survey participants compared with all non-responders, but for the subset of people who did not respond due to illness there were much greater differences in health service use [43]. Similar to our study, the one study examining associations between demographic factors and health service use (including use of prescription drugs, hospitalisations, specialist, allied and dental care) among responders to a health survey found estimates were similar to those measured from target sample [10]. Our study complements another study on the representativeness of 45 and Up Study cohort which demonstrated the generalisability of aetiological associations measured from 45 and Up Study participants to survey-based NSW population estimates [31].

\section{Conclusions}

This study contributes to the empirical evidence base for generalising health service use associations measured from non-representative samples. There was little evidence of bias in risk factor associations for the cancers and outcomes examined. However, the comparability of participants and non-participants with respect to the risk factor measure requires consideration. Further study is warranted on health service use in the primary and outpatient settings since the potential for selection bias is greater.

\section{Additional file}

Additional file 1: Socioeconomic position by rurality and univariable and multivariable models of health service use outcomes. The additional file contains ratios of relative frequencies for area-based socioeconomic position stratified by rurality (major city; regional and remote) and univariable and multivariable logistic regression models of health service use outcomes (resection; > 4 weeks in hospital; > 2 emergency department attendances; one-year all-cause post-diagnosis survival) for 45 and Up Study participants and NSW residents aged $\geq 45$ years at diagnosis of bowel or lung cancer. (DOCX $610 \mathrm{~kb}$ )

\section{Abbreviations}

CL: Confidence limit; ED: Emergency department; ICD-10-AM: International Classification of Diseases, 10th Edition, Australian Modification; NSW: New South Wales; NSWCR: NSW Cancer Registry; ROR: Relative odds ratio; RRF: Ratio of relative frequency

\section{Acknowledgements}

This research was completed using data collected through the 45 and Up Study (www.saxinstitute.org.au). The 45 and Up Study is managed by the Sax Institute in collaboration with major partner Cancer Council NSW; and partners: the National Heart Foundation of Australia (NSW Division); NSW Ministry of Health; NSW Government Family \& Community Services - Ageing, Carers and the Disability Council NSW; and the Australian Red Cross Blood Service. We thank the many thousands of people participating in the 45 and Up Study.

\section{Funding}

This research did not receive any specific grant.

\section{Availability of data and materials}

The data that support the findings of this study are available from the relevant data custodians of the study datasets. The 45 and Up Study data were used under license for the current study. Restrictions by the data custodians mean that the data are not publicly available or able to be provided by the authors.

Researchers wanting to access the datasets used in this study should refer to the 45 and Up Study application process (www.saxinstitute.org.au/our-work) 45-up-study/for-researchers/) and the Centre for Health Record Linkage application process (www.cherel.org.au/apply-for-linked-data).

\section{Authors' contributions}

$J Y$ and MS conceived the experiment. NC designed the experiment with input on the study protocol from all authors. RW provided advice on statistical analysis. NC conducted the analyses and drafted the manuscript. SP conducted secondary data analysis. DB provided input into the interpretation of the data. All authors provided critical revision of the manuscript and approved the final version.

\section{Ethics approval and consent to participate}

The study was conducted with ethical approval from the NSW Population and Health Services Research Ethics Committee (HREC/14/CIPHS/60). The 45 and Up Study is approved by the University of New South Wales Ethics Committee. Participants of the 45 and Up Study provided written consent to the linkage of their health-related records.

\section{Consent for publication}

Not applicable.

\section{Competing interests}

NC, SP, MS, RW and JY declare that they have no competing interests. DB was an employee of the Sax Institute NSW and Research Manager of the 45 and Up Study for part of the time this study was being conducted.

\section{Publisher's Note}

Springer Nature remains neutral with regard to jurisdictional claims in published maps and institutional affiliations.

\section{Author details}

${ }^{1}$ Cancer Institute NSW, PO Box 41, Alexandria, Sydney, NSW 1435, Australia. ${ }^{2}$ Asbestos Diseases Research Institute, Sydney, Australia. ${ }^{3}$ Sax Institute, Sydney, Australia. ${ }^{4}$ School of Public Health, University of Sydney and Sydney Local Health District, Sydney, Australia.

Received: 4 May 2018 Accepted: 27 June 2018

Published online: 08 August 2018

\section{References}

1. Browning $\mathrm{CJ}$, Kendig $\mathrm{H}$. Cohort profile: the Melbourne longitudinal studies on healthy ageing program. Int J Epidemiol. 2010;39:e1-7.

2. Huisman M, Poppelaars J, van der Horst M, Beekman AT, Brug J, van Tilburg TG, et al. Cohort profile: the longitudinal aging study Amsterdam. Int J Epidemiol. 2011;40:868-76.

3. Steptoe A, Breeze E, Banks J, Nazroo J. Cohort profile: the English longitudinal study of ageing. Int J Epidemiol. 2013;42:1640-8.

4. Martin S, Haren M, Taylor A, Middleton S, Wittert G. Members of the Florey Adelaide male ageing study. Cohort profile: the Florey Adelaide male ageing study (FAMAS). Int J Epidemiol. 2007:36:302-6.

5. Kearney PM, Cronin H, O'Regan C, Kamiya Y, Savva GM, Whelan B, et al. Cohort profile: the Irish longitudinal study on ageing. Int J Epidemiol. 2011; 40:877-84.

6. Schooling C, Chan W, Leung S, Lam T, Lee S, Shen C, et al. Cohort profile: Hong Kong Department of Health elderly health service cohort. Int J Epidemiol. 2016;45:64-72.

7. Zhao Y, Hu Y, Smith JP, Strauss J, Yang G. Cohort profile: the China health and retirement longitudinal study (CHARLS). Int J Epidemiol. 2014;43:61-8.

8. Wong R, Michaels-Obregon A, Palloni A. Cohort profile: the Mexican health and aging study (MHAS). Int J Epidemiol. 2017;46:e2.

9. Rothman KJ, Gallacher JE, Hatch EE. Why representativeness should be avoided. Int J Epidemiol. 2013;42:1012-4. 
10. Reijneveld SA, Stronks K. The impact of response bias on estimates of health care utilization in a metropolitan area: the use of administrative data. Int J Epidemiol. 1999;28:1134-40.

11. Lamers LM. Medical consumption of respondents and non-respondents to a mailed health survey. Eur J Pub Health. 1997;7:267-71.

12. Drivsholm T, Eplov LF, Davidsen $M$, Jørgensen $T$, Ibsen $H$, Hollnagel $H$, et al. Representativeness in population-based studies: a detailed description of non-response in a Danish cohort study. Scand J Public Health. 2006;34:623-31.

13. Osler M, Schroll M. Differences between participants and non-participants in a population study on nutrition and health in the elderly. Eur J Clin Nutr. 1992;46:289-95.

14. Banks E, Redman S, Jorm L, Armstrong B, Bauman A, Beard J, et al. Cohort profile: the 45 and up study. Int J Epidemiol. 2008;37:941-7.

15. Australian Institute of Health and Welfare. Australian Burden of Disease Study: impact and causes of illness and death in Australia 2011. Australian Burden of Disease Study series no. 3. BOD 4. Canberra: AlHW; 2016.

16. Global Burden of Disease Cancer Collaboration, Fitzmaurice C, Dicker D, Pain A, Hamavid H, Moradi-Lakeh M, et al. The global burden of cancer 2013. JAMA Oncol. 2015;1:505-27.

17. Farmer P, Frenk J, Knaul FM, Shulman LN, Alleyne G, Armstrong L, et al. Expansion of cancer care and control in countries of low and middle income: a call to action. Lancet. 2010;376:1186-93.

18. Forrest LF, Adams J, Wareham H, Rubin G, White M. Socioeconomic inequalities in lung cancer treatment: systematic review and meta-analysis. PLoS Med. 2013;10:e1001376

19. Aarts MJ, Lemmens VEPP, Louwman MWJ, Kunst AE, Coebergh JWW. Socioeconomic status and changing inequalities in colorectal cancer? A review of the associations with risk, treatment and outcome. Eur J Cancer. 2010;46:2681-95.

20. Australian Bureau of Statistics. 4390.0 Private Hospitals, Australia 2011-12. Canberra: ABS; 2013.

21. Australian Institute of Health and Welfare. Australian hospital statistics 2011-12. Health services series no. 50. Cat. no. HSE 134. Canberra: AlHW; 2013.

22. Australian Institute of Health and Welfare. Cancer in Australia 2017. Cancer series no. 101. Cat. no. CAN 100. Canberra: AlHW; 2017.

23. Goldsbury DE, O'Connell DL, Girgis A, Wilkinson A, Phillips JL, Davidson PM, et al. Acute hospital-baseColombet M, Mery L, Piñeros M, Znaor A, Zanetti R, Ferlay Jd services used by adults during the last year of life in New South Wales, Australia: a population-based retrospective cohort study. BMC Health Serv Res. 2015;15:537.

24. Australian Bureau of Statistics. 2039.0 - Information Paper: An Introduction to Socio-Economic Indexes for Areas (SEIFA), Australia, 2006. Canberra: ABS; 2008.

25. Australian Bureau of Statistics. 1216.0.15.003 - Australian Standard Geographical Classification (ASGC) Remoteness Area Correspondences. Canberra: ABS; 2011

26. Quan H, Li B, Couris CM, Fushimi K, Graham P, Hider P, et al. Updating and validating the Charlson comorbidity index and score for risk adjustment in hospital discharge abstracts using data from 6 countries. Am J Epidemiol. 2011;173:676-82.

27. Nilsen RM, Vollset SE, Gjessing HK, Skjaerven R, Melve KK, Schreuder P, et al. Self-selection and bias in a large prospective pregnancy cohort in Norway. Paediatr Perinat Epidemiol. 2009;23:597-608.

28. Nohr EA, Frydenberg M, Henriksen TB, Olsen J. Does low participation in cohort studies induce bias? Epidemiology. 2006;17:413-8.

29. Bray F, Colombet M, Mery L, Piñeros M, Znaor A, Zanetti R, Ferlay J, editors Cancer Incidence in Five Continents, Vol. XI (electronic version). Lyon: International Agency for Research on Cancer; 2017. http://ci5.iarc.fr. Accessed 3 Mar 2018.

30. Creighton N, Perez D, Cotter T. Smoking-attributable cancer mortality in NSW, Australia, 1972-2008. Public Health Res Pract. 2015;25:e2531530.

31. Mealing NM, Banks E, Jorm LR, Steel DG, Clements MS, Rogers KD. Investigation of relative risk estimates from studies of the same population with contrasting response rates and designs. BMC Med Res Methodol. 2010;10:26.

32. Sax Institute. The 45 and Up Study Baseline Questionnaire Data Book. Sydney: Sax Institute; 2011. www.saxinstitute.org.au/our-work/45-up-study/ data-book. Accessed 3 Mar 2018.
33. Centre for Epidemiology and Evidence. HealthStats NSW: Smoking status in adults by age, category and year. www.healthstats.nsw.gov.au. Accessed 3 Mar 2018.

34. Gilthorpe MS, Wilson RC. Rural/urban differences in the association between deprivation and healthcare utilisation. Soc Sci Med. 2003;57:2055-63.

35. Hernan MA, Hernandez-Diaz S, Robins JM. A structural approach to selection bias. Epidemiology. 2004;15:615-25.

36. Sørensen K, Van den Broucke S, Fullam J, Doyle G, Pelikan J, Slonska Z, et al, Health literacy and public health: a systematic review and integration of definitions and models. BMC Public Health. 2012;12:80.

37. Babitsch B, Gohl D, von Lengerke T. Re-revisiting Andersen's behavioral model of health services use: a systematic review of studies from 19982011. Psychosoc Med 2012;9:Doc11.

38. Rothman K, Greenland S, Lash T. Validity in epidemiologic studies. In: Rothman K, Greenland S, Lash T, editors. Modern Epidemiology. 3rd ed. Philadelphia: Lippincott Williams \& Wilkins; 2008.

39. Macleod U, Mitchell ED, Burgess C, Macdonald S, Ramirez AJ. Risk factors for delayed presentation and referral of symptomatic cancer: evidence for common cancers. Br J Cancer. 2009;101:S92-S101.

40. Smith LK, Pope C, Botha JL. Patients' help-seeking experiences and delay in cancer presentation: a qualitative synthesis. Lancet. 2005;366:825-31.

41. Willett WC, Blot WJ, Colditz GA, Folsom AR, Henderson BE, Stampfer MJ. Merging and emerging cohorts: not worth the wait. Nature. 2007:445:257-8.

42. Greenland S, Mansournia MA, Altman DG. Sparse data bias: a problem hiding in plain sight. BMJ. 2016;352:11981.

43. Gundgaard J, Ekholm O, Hansen EH, Rasmussen NK. The effect of nonresponse on estimates of health care utilisation: linking health surveys and registers. Eur J Pub Health. 2008;18:189-94.

44. Rinne ST, Wong ES, Lemon JM, Perkins M, Bryson CL, Liu CF. Survey nonresponders incurred higher medical utilization and lower medication adherence. Am J Manag Care. 2015;21:e1-8.

\section{Ready to submit your research? Choose BMC and benefit from:}

- fast, convenient online submission

- thorough peer review by experienced researchers in your field

- rapid publication on acceptance

- support for research data, including large and complex data types

- gold Open Access which fosters wider collaboration and increased citations

- maximum visibility for your research: over 100M website views per year

At BMC, research is always in progress.

Learn more biomedcentral.com/submissions 